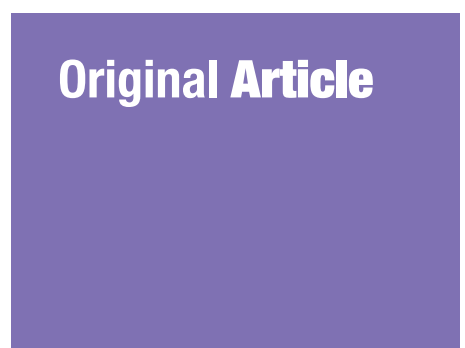

Submitted: 23 Aug 2015

Accepted: 7 Aug 2016

Online: 7 Dec 2016

\section{The Effects of Crocin on 6-0HDA-Induced Oxidative/Nitrosative Damage and Motor Behaviour in Hemiparkinsonian Rats}

\author{
Maryam Hosseini ${ }^{1}$, Ziba Rajaei ${ }^{1,2}$, Hojjatallah Alaei ${ }^{3}$, \\ Mohamadhasan TAJADINI ${ }^{4}$
}
1 Department of Physiology, School of Medicine, Isfahan University of Medical Sciences, Isfahan, Iran
$2 \quad$ Neurocognitive Research Center, School of Medicine, Mashhad University of Medical Sciences, Mashhad, Iran
3 Isfahan Neurosciences Research Center, Alzahra Hospital, Isfahan University of Medical Sciences, Isfahan, Iran
$4 \quad$ Physiology Research Center, Isfahan University of Medical Sciences, Isfahan, Iran

To cite this article: Hosseini M, Rajaei Z, Alaei H, Tajadini M. The effects of crocin on 6-OHDA-induced oxidative/ nitrosative damage and motor behaviour in hemiparkinsonian rats. Malays $J$ Med Sci. 2016;23(6):35-43. http://dx.doi.org/10.21315/mjms2016.23.6.4

To link to this article: http://dx.doi.org/10.21315/mjms2016.23.6.4

\title{
Abstract
}

Background: Crocin is considered to prevent oxidative stress-related diseases, such as ischemia and Alzheimer's. The aim of the present investigation was to evaluate the effects of crocin on motor behaviour and 6-OHDA-induced oxidative/nitrosative damage to the striatum in an experimental model of Parkinson's disease.

Methods: Left medial forebrain bundle was lesioned by microinjection of 6-OHDA (16 $\mu \mathrm{g}$ in $0.2 \%$ ascorbate-saline). Crocin (30 and $60 \mathrm{mg} / \mathrm{kg}$ ) was injected intraperitoneally three days before surgery until six weeks. Rotational behaviour and biochemical analysis were used to evaluate the effect of crocin in a unilateral 6-OHDA-induced model of Parkinson's disease.

Results: The contralateral rotations induced by apomorphine in 6-OHDA lesioned group were highly significant $(P<0.001)$ as compared to the sham group. Moreover, chronic administration of crocin at doses of 30 and $60 \mathrm{mg} / \mathrm{kg}$ over six weeks did not change the rotations. The TBARS and nitrite levels in the striatum were also significantly $(P<0.05)$ increased in lesioned group. Treatment with crocin at a dose of $60 \mathrm{mg} / \mathrm{kg}$ significantly decreased the nitrite levels $(P<0.05)$ in the striatum.

Conclusion: Crocin at a dose of $60 \mathrm{mg} / \mathrm{kg}$ could be effective in preventing the nitrosative damage in the striatum. Further investigations using higher doses of crocin is suggested to get the full neuroprotective effects of crocin in Parkinson's disease.

Keywords: crocin, 6-Hydroxydopamine, oxidative stress, nitric oxide, motor activity, Parkinson disease

\section{Introduction}

Parkinson's disease (PD) is an age-related movement disorder that affects on $1 \%$ of the people aged over 65 years (1). The degeneration of nigrostriatal dopaminergic neurons, which is accompanied by reduction of dopamine in the striatum, is the neuropathological basis of the movement disorders in the PD (2). The clinical symptoms of PD manifests when about $50 \%$ of nigrostriatal dopaminergic neurons and $70 \%$ of striatal dopaminergic axons are lost (3). The mechanisms responsible for dopaminergic neuronal loss in $\mathrm{PD}$ are complex and remain to be completely determined. However, several pathogenic factors including; oxidative 
and nitrosative stress (4-6), mitochondrial dysfunction (7), apoptosis (8), inflammatory responses (9) and excitotoxicity have been proposed for the degeneration of dopaminergic neurons.

Compelling evidence implicates that increased reactive oxygen species (ROS) and oxidative damage are involved in the cascade of events leading to degeneration of dopaminergic neurons $(10,11)$. This is mainly due to the observations that increased level of lipid peroxidation $(4,12)$, modifications of proteins (13), and DNA and RNA oxidation products (14) are seen in the brain of parkinsonian patients.

It is also believed that reactive nitrogen species (RNS), such as nitric oxide (NO) and peroxynitrite, play a major role in the toxicity of nigrostriatal dopaminergic neurons in PD $(15,16)$. NO is synthesised from L-arginine by the enzymes nitric oxide synthases (NOS) (17). Under pathological conditions, production of NO is mainly stimulated by activation of inducible NOS (iNOS) (18). Induction of iNOS produces high levels of NO which leads to neurotoxicity by production of peroxynitrite (19). Peroxynitrite, which is a more active and potent oxidizing agent than NO, can induce DNA fragmentation and lipid peroxidation (20). It has been reported that neurodegeneration in PD is associated with increase in iNOS expression in the substantia nigra $(21,22)$. It also has been shown that inhibition of iNOS expression with the inhibitors of NOS protects neurons from degeneration in PD animal models (23).

Crocin, crocetin digentiobiose ester, is a known potent antioxidant found in the stigma of Crocus sativus. A wide range of pharmacological activities has been reported for crocin, including; antioxidant $(24,25)$, anticancer (26), antiinflammatory (27, 28), antihyperglycemic (29), hypolipidemic (30), anti-atherosclerotic (31) and hepatoprotective effects (29). The antioxidant activity and radical scavenging properties of crocin has been demonstrated in neurodegenerative diseases such as Alzheimer's (32) and cerebral ischemia (33). It also has been reported that treatment with crocin decreased NO levels and NOS activity in cortical microvascular homogenates in an ischemic model in rat (33). Moreover, crocin inhibited the lipopolysaccaride-induced NO release from cultured rat brain microglial cells (27).

Based on the mentioned evidence, we decided to evaluate the effects of chronic treatment with crocin at doses of 30 and $60 \mathrm{mg} /$ $\mathrm{kg}$ over six weeks on oxidative and nitrosative damage and motor behaviour in a 6-OHDA model of PD.

\section{Materials and Methods}

\section{Animals}

Experiments were performed using male Wistar rats weighing 250-30og. Animals were maintained at $22^{\circ} \mathrm{C} \pm 2^{\circ} \mathrm{C}$ on a standard pellet diet and tap water ad libitum. The Ethic Committee for Animal Experiments at Isfahan University of Medical Sciences approved the study and all experiments were conducted in accordance with the National Institute of Health Guide for the Care and Use of Laboratory Animals (NIH Publications $\mathrm{N}^{\mathrm{O}}$ 8023, revised 1996).

\section{Experimental design}

The animals were randomly assigned to four groups, with eight rats in each group, as follows:

1. Group 1: Normal saline sham-operated group (0.2\% ascorbate-saline into the left medial forebrain bundle (MFB), saline ip)

2. Group 2: Normal saline-treated lesioned

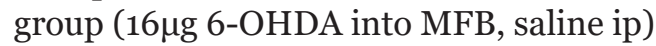

3. Group 3: Crocin-treated lesioned group

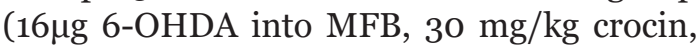
ip)

4. Group 4: Crocin-treated lesioned group (16ug 6-OHDA into MFB, $60 \mathrm{mg} / \mathrm{kg}$ crocin, ip)

Crocin at doses of 30 and $60 \mathrm{mg} / \mathrm{kg}$ or normal saline were injected intraperitoneally three days before the surgery for six weeks.

The rats were anaesthetised with chloral hydrate (450 mg/kg, ip) (34) and then placed in a stereotaxic apparatus. The scalp was cleaned with an iodine solution and lidocaine was injected ( $2 \%$ solution, $\mathrm{Sc}$ ). A midline skin incision was made with subsequent drilling of the skull. 6-OHDA (16 $\mu \mathrm{g} / 4 \mu \mathrm{l} 0.2 \%$ ascorbatesaline) was injected into the left MFB by a Hamilton microsyringe according to the coordinates: AP: $-4.5 \mathrm{~mm}$; ML: $-1.7 \mathrm{~mm}$; DV: $-8.2 \mathrm{~mm} \mathrm{(35).} \mathrm{The} \mathrm{rats} \mathrm{of} \mathrm{sham-operated}$ group also received an identical volume of the ascorbate-saline as vehicle. The injection rate was $1 \mu \mathrm{l} / \mathrm{min}$ and the needle was kept in place for an additional $5 \mathrm{~min}$ before being slowly retracted. After surgery, all rats were treated with penicillin ( $0.2 \mathrm{ml}$, ip), placed singly into a clean 
page and kept warm until complete recovery occurred.

At the end of the experiment, the animals were sacrificed and the striatum was dissected out, washed immediately in ice-cold saline, and homogenised in the $\mathrm{NaCl} 0.9 \%$ solution by a homogeniser.

\section{Apomorphine-induced rotations}

The hemiparkinsonian rats were diagnosed by observing the rotational behaviour after injection of apomorphine hydrochloride (SigmaAldrich, USA) at the end of the 2nd, 4th and 6th week after surgery. Apomorphine hydrochloride was dissolved in normal saline and injected intraperitoneally at a dose of $2 \mathrm{mg} / \mathrm{kg}$. On day test, the animals were allowed to habituate to a transparent plexiglass container $(28 \mathrm{~cm} \times$ $28 \mathrm{~cm} \times 50 \mathrm{~cm}$ ) for $10 \mathrm{~min}$. One minute after the injection of apomorphine, full rotations were counted at $10 \mathrm{~min}$ intervals for $60 \mathrm{~min}$ in a dimly-lit and quiet room. The number of ipsilateral rotations was counted as positive scores and that of contralateral rotations as negative scores. Net number of rotation was defined as the difference between the rotations in both directions (36).

\section{Lipid peroxidation levels}

The lipid peroxidation level of the striatum was measured by thiobarbituric acid reactive substance (TBARS) assay. Briefly, a mixture of trichloroacetic acid, thiobarbituric acid, and $\mathrm{HCl}$ were added to $1 \mathrm{~mL}$ of homogenate, and the mixture was heated for $45 \mathrm{~min}$ in a boiling water bath. After cooling, the samples were centrifuged at $1000 \times \mathrm{g}$ for $10 \mathrm{~min}$ and the absorbance was measured at $535 \mathrm{~nm}$. The level of TBARS was calculated by: $\mathrm{C}(\mathrm{M})=\mathrm{A} / 1.65 \times 10^{5}(37)$.

\section{Glutathion peroxidase assay}

Glutathion peroxidase activity was measured with the glutathion peroxidase assay kit (Cat. No. RS505, Randox Labs, UK).

\section{Nitrite levels}

The level of nitrite was measured using a colorimetric assay kit (Promega Corporation, USA) that involved the Griess reaction. Briefly, the samples were incubated with sulphanilamide solution and then the N-(1-naphthyl) ethylenediamine solution was added. Then, the absorbance was measured by a microreader in the wave length of $492 \mathrm{~nm}$. The nitrite concentration of samples was measured using the nitrite standard curve.

\section{Statistical analysis}

Behavioral data were analysed by twoway repeated measures ANOVA with groups as between-subject factor and time as within subject factor. Then, one-way ANOVA was performed for comparing the groups in each week followed by Tukey post hoc test for multiple comparisons. Biochemical data were analysed by one-way ANOVA followed by Tukey post hoc test. $P<0.05$ was considered statistically significant. The results are presented as the mean \pm SEM.

\section{Results}

\section{Effects of crocin on rotational behavior}

As shown in Figure 1, administration of apomorphine hydrochloride to 6-OHDA-lesioned rats produced contralateral rotations towards the lesion side at the end of the 2nd, 4th and 6th week after surgery, indicating unilateral damage to the left striatum. No such rotations were observed in sham group rats.

Analysing data with two-way repeated measures ANOVA revealed a significant difference between groups $(P<0.001)$ and within groups in different weeks $(P<0.001)$. Then, for comparison of various groups in each week, one-way ANOVA was performed followed by Tukey post hoc test. The ANOVA analysis showed that the number of contralateral rotations in 6-OHDA-lesioned group and crocintreated lesioned groups were significantly different from sham group $(P<0.001)$ for each week (Figure 1).

\section{Effects of crocin on lipid peroxidation levels}

Injection of 6-OHDA resulted in significant elevation of TBARS levels $(P<0.05)$ in the striatum. Moreover, the results showed that treatment of lesioned rats with crocin at doses of 30 and $60 \mathrm{mg} / \mathrm{kg}$ for six weeks did not change the increased TBARS levels in the striatum (Figure 2).

\section{Effects of crocin on nitrite levels}

The nitrite levels of the striatum were significantly $(P<0.05)$ increased in the 6-OHDA-lesioned animals as compared to the sham group. In addition, a significant decrease in nitrite levels was observed in the 6-OHDAlesioned rats treated with crocin at a dose of $60 \mathrm{mg} / \mathrm{kg}$ as compared to the lesioned group $(P<0.05$, Figure 3$)$. 


\section{Effects of crocin on glutathion peroxidase activity}

The results showed that there was no significant difference in glutathione peroxidase activity in the striatum of sham, 6-OHDAlesioned rats and lesioned groups treated with crocin at doses of 30 and $60 \mathrm{mg} / \mathrm{kg}$ at the end of week 6 (Figure 4).

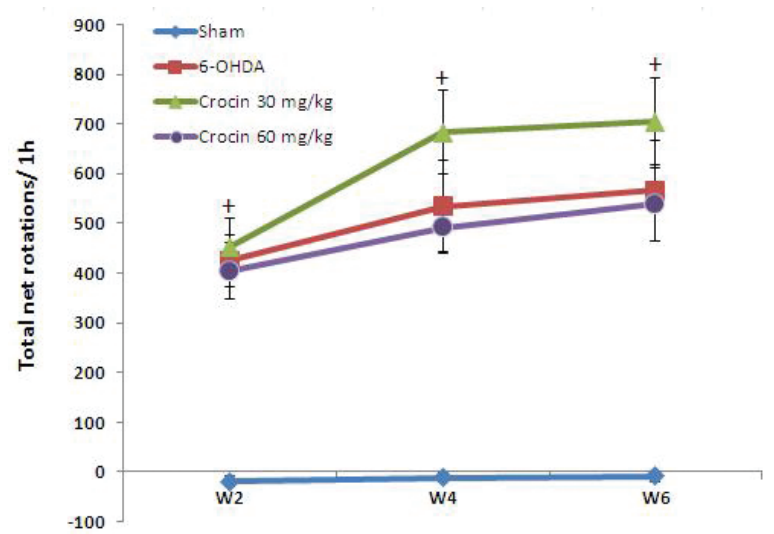

Figure 1. Apomorphine-induced rotations (mean $\pm \mathrm{SEM}$ ) in the sham, 6-OHDAlesioned group and crocin-treated lesioned groups at the end of the 2nd, 4th and 6th week after surgery. Crocin was administered ip daily at doses of 30 and $60 \mathrm{mg} / \mathrm{kg}$ for six weeks. $+P<0.001$ vs sham group by Tukey post hoc.

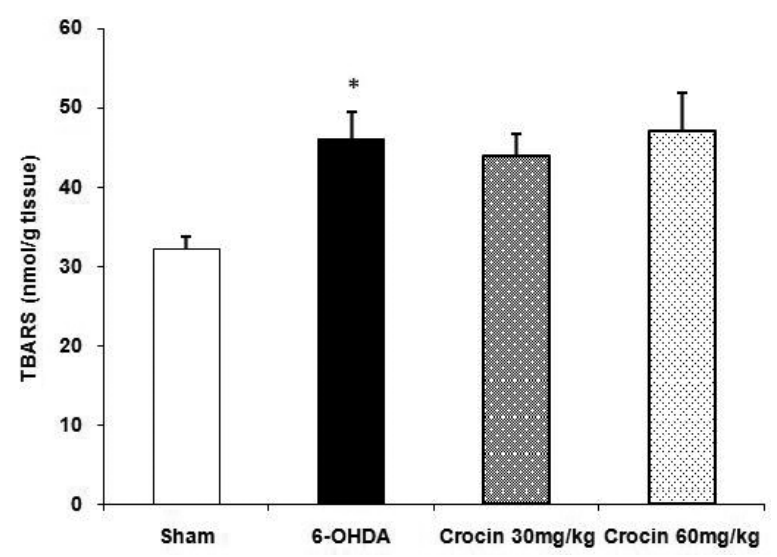

Figure 2. Effect of crocin on lipid peroxidation levels (mean \pm SEM) in the striatum of the sham and experimental groups. Crocin was administered daily at doses of 30 and $60 \mathrm{mg} / \mathrm{kg}$ for six weeks. ${ }^{*} P<0.05$ vs sham group.

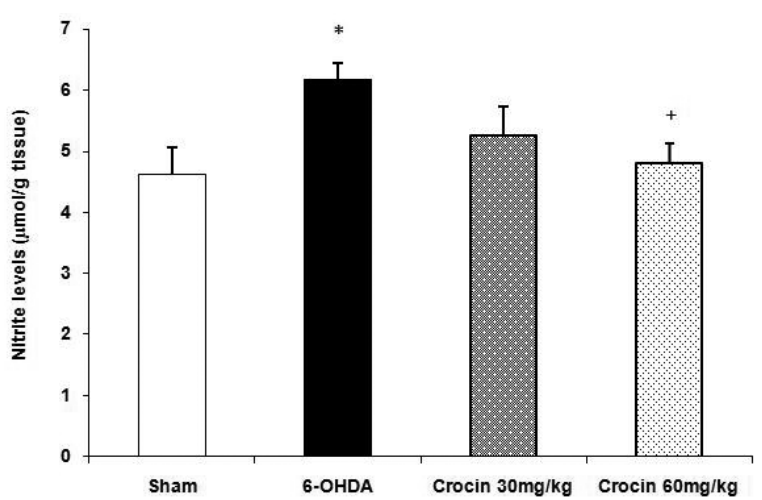

Figure 3. Effects of crocin on nitrite levels (mean \pm SEM) in the striatum of the sham and experimental groups. Crocin was administered daily at doses of 30 and $60 \mathrm{mg} / \mathrm{kg}$ for six weeks. ${ }^{*} P<0.05$ vs sham group, $+P<0.05$ vs 6 -OHDA-lesioned group.

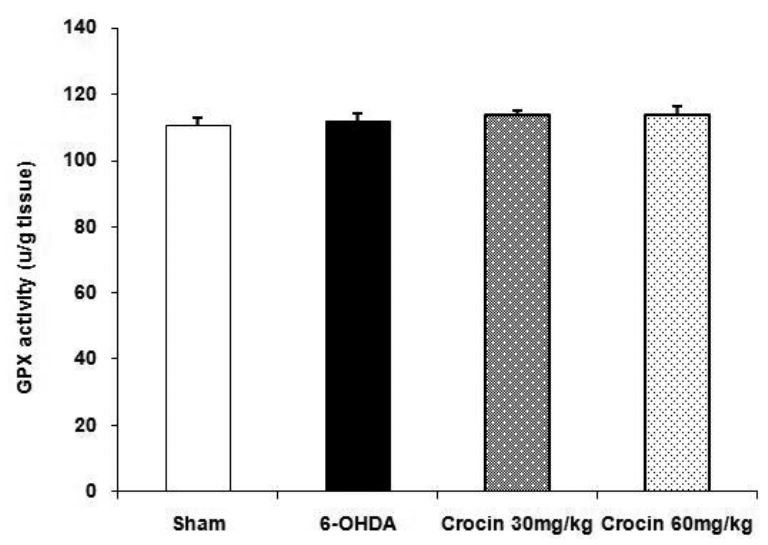

Figure 4. Effects of crocin on glutathione peroxidase activity (mean \pm SEM) in the striatum of the sham and experimental groups. Crocin was administered daily at doses of 30 and $60 \mathrm{mg} / \mathrm{kg}$ for six weeks.

\section{Discussion}

This study demonstrated that the microinjection of 6-OHDA into the MFB resulted in motor deficits accompanying oxidative and nitrosative damage to the striatum. The MFB is a tract containing fibers from brainstem regions, including the substantia nigra and ventral tegmental area, as well as fibers from the basal olfactory regions, the periamygdaloid region and the septal nuclei. Unilateral injection of 
6-OHDA into the MFB can cause the destruction of dopaminergic neurons in the substantia nigra (A9) and ventral tegmental area (A10) (38). The axons of nigrostriatal dopaminergic neurons run along the MFB, terminate in the dorsal striatum and participates in motor system. In addition to depleting the nigrostriatal dopamine pathway, MFB lesions also deplete the dopaminergic neurons of the ventral tegmental area, which project to the nucleus accumbens and participates in reward system (39).

The unilateral lesion of the nigrostriatal dopaminergic system by 6-OHDA decreases dopamine level in the striatum and upregulates dopamine postsynaptic receptors at the same side. These changes produce a functional and motor asymmetry that can be assessed by dopamine agonists such as apomorphine (40). Apomorphine is a dopamine receptor agonist which can stimulate both types of dopamine receptors (Dl, D2) and can therefore directly affect presynaptic and postsynaptic receptors. Apomorphine induces rotations contralateral to the lesioned side because of changes in dopamine receptor mechanisms, especially due to the stimulation of upregulated D2 receptors in the denervated striatum (41). Contralateral rotations induced by apomorphine in 6-OHDA-lesioned rats is a reliable marker for the nigrostriatal dopamine depletion (42). In the present study, injection of apomorphine to 6-OHDA group rats caused significant contralateral rotations, which happens after degeneration of at least $75 \%$ of nigrostriatal dopaminergic neurons (43). Furthermore, the administration of 30 and 60 $\mathrm{mg} / \mathrm{kg}$ of crocin for six weeks did not decrease the apomorphine-induced rotations in rats.

6-OHDA is a neurotoxin that could easily undergo autoxidation to give hydrogen peroxide and superoxide radicals, which finally leads to production of hydroxyl free radicals (44). In the present study, injection of 6-OHDA into the MFB caused oxidative damage to membrane lipids, as evidenced by increased levels of TBARS in the striatum at the end of week 6 . This oxidative neuronal damage in 6-OHDA-lesioned animals is in agreement with previous reports $(45,46)$. In fact, our results confirm that the neurotoxin through the formation of ROS initiated an oxidative cascade of events in the striatum, which finally led to membrane damage.

However, our results also showed that chronic treatment with crocin at doses of 30 and $60 \mathrm{mg} / \mathrm{kg}$ did not attenuate the elevated levels of TBARS in the striatum. This is in contrary with previous studies which report the protective effects of crocin in oxidative insults such as ischemia-reperfusion (33), diabetes (29) and Alzheimer's (32). The reasons for this discrepancy between our results and previous studies are not known. Nonetheless, a possible answer could be related to its dosage and duration of treatment. We suggest that initial oxidative stress might be scavenged or attenuated by the antioxidant capability of crocin at applied doses; however, when there is substantial ongoing oxidative stress (47)-as in the 6-OHDA model of PD-the antioxidant response wanes or is overwhelmed over time, and at that point, crocin could not act as an antioxidant.

However, Parkinson's disease is a multifactorial neurodegenerative disease and nitrergic mechanisms could also contribute to the dopamine neuronal death seen in PD (48). The administration of 6-OHDA induces microglial activation, leading to the production of cytokines and free radicals, including NO (3). NO is a short-lived free radical, which acts as a precursor for a few chemical substances and induce nitrosative stress. Despite inorganic and gaseous nature, NO is reported to be one of the main malefactors of PD (49). NO gets converted into nitrite in a few seconds that is relatively more stable (50). The roles of NO in the biological functions are mostly inferred either via the quantitative evaluation of nitrite or via the expression levels of NOSs (51).

6-OHDA is found to increase the nitrite levels and iNOS expression in rats $(21,48)$. It has been reported that induction of iNOS and NO by 6-OHDA deplete dopaminergic cells in the substantia nigra, and dopamine and its metabolites in the striatum, and pretreatment with LNAME (NOS inhibitor) reverses these adverse effects $(21,52)$. It also has been shown that NOS inhibitor 7-nitroindazole rescues from 6-OHDA-induced dopaminergic neurodegeneration (3).

The present findings showed that 6-OHDA increased the levels of the nitrite, stable NO metabolite, in the striatum of lesioned rats. This is consistent with previous studies which report the increased levels of nitrite in the midbrain and striatum of 6-OHDA lesioned rats $(21,53)$. The results also showed that chronic treatment with crocin significantly decreased the nitrite levels in this tissue. Along with this, it has been reported that crocin inhibited the lipopolysaccarideinduced NO release from cultured rat brain microglial cells (27). Moreover, treatment with crocin decreased NO levels and NOS activity 
in cortical microvascular homogenates in an ischemic model in rat (33). Collectively, it seems that crocin could be effective in preventing the nitrosative stress in the striatum in a 6-OHDA model of PD.

\section{Conclusion}

Our findings demonstrated that chronic treatment with crocin at a dose of $60 \mathrm{mg} / \mathrm{kg}$ for six weeks could be effective in preventing nitrosative stress in hemiparkinsonian rats. Nonetheless, it had no effect on oxidative stress and could not improve apomorphine-induced rotations. Regarding that the oxidative and nitrosative stress caused by accumulation of ROS and RNS can affect different pathways and contribute to the pathogenesis of PD (6), our study further supports the notion that oxidative stress play a major role in 6-OHDA-induced parkinsonism. Further investigations using higher doses of crocin is suggested in order to gain access to full neuroprotective effects of crocin in Parkinson's disease.

\section{Acknowledgement}

The results presented in this work have been taken from a student's thesis. This study was supported by Isfahan University of Medical Sciences.

\section{Conflict of interest}

None.

\section{Funds}

I confirm that I have mentioned all organisations that funded my research in the acknowledgements section of my submission where appropriate.

\section{Authors' Contribution}

Conception and design: ZR, HA

Analysis and interpretation of the data: ZR

Drafting of the article: ZR

Critical revision of the article for important intellectual content: ZR, HA

Final approval of the article: ZR, HA

Administrative, technical, or logistic support: MH, MT

Collection and assembly of data: $\mathrm{MH}$

\section{Correspondence}

Dr. Ziba Rajaei

PhD (Isfahan University of Medical Sciences)

Department of Physiology

School of Medicine

Isfahan University of Medical Sciences

Isfahan, Iran

Tel: 98-31-37929181

Fax: 98-31-36688597

E-mail: rajaeiz@med.mui.ac.ir

\section{References}

1. Reeve A, Simcox E, Turnbull D. Ageing and Parkinson's disease: why is advancing age the biggest risk factor? Ageing Res Rev. 2014;14:1930. http://dx.doi.org/10.1016/j.arr.2014.01.004.

2. Hornykiewicz O, Kish SJ. Biochemical pathophysiology of Parkinson's disease. Adv Neurol. 1987;45:19-34.

3. Yuste JE, Echeverry MB, Ros-Bernal B, Gomez A, Ros CM, Campuzano CM, et al. 7-Nitroindazole down-regulates dopamine/ DARPP-32 signaling in neostriatal neurons in a rat model of Parkinson's disease. Neuropharmacology. 2012;63(7):1258-1267. http://dx.doi.org/10.1016/j.neuropharm.2012. 07.031.

4. Jenner P. Oxidative stress in Parkinson's disease. Ann Neurol. 2003;53(Suppl 3):S26-S38.

5. Kavya R, Saluja R, Singh S, Dikshit M. Nitric oxide synthase regulation and diversity: Implications in Parkinson's disease. Nitric Oxide. 2006;15(4):280-294.

6. Tsang AHK, Chung KKC. Oxidative and nitrosative stress in Parkinson's disease. Biochimica et Biophysica Acta. 2009;1792(7):643-650. http://dx.doi.org/ 10.1016/j.bbadis.2008.12.006.

7. Jenner P. Altered mitochondrial function, iron metabolism and glutathione levels in Parkinson's disease. Acta Neurol Scand. Suppl 1993;146:613.

8. Ziv I, Melamed E, Nardi N. Role of apoptosis in the pathogenesis of Parkinson's disease: A novel therapeutic opportunity. Mov Disord. 1998;13(6):865-870. 
9. Hirsch EC, Hunot S, Hartmann A. Neuroinflammatory processes in Parkinson's disease. Parkinsonism Relat Disord. 2005;11(Suppl 1):9-15.

10. Jenner $\mathrm{P}$, Olanow $\mathrm{W}$. The pathogenesis of cell death in Parkinson's disease. Neurol. 2006;66(10 Suppl 4):24-36. http://dx.doi.org/10.1212/ WNL.66.10_suppl_4.S24

11. Dias V, Junn E, Mouradian MM. The role of oxidative stress in Parkinson's disease. $J$ Parkinsons Dis. 2013;3(4):461-91. http://dx.doi. org/10.3233/JPD-130230.

12. Yoritaka A, Hattori N, Uchida K, Tanaka M, Stadtman E, Mizuno Y. Immunohistochemical detection of 4-hydroxynonenal protein adducts in Parkinson disease. Proc Natl Acad Sci USA. 1996;93(7):2696-2701.

13. Floor E, Wetzel M. Increased protein oxidation in human substantia nigra pars compacta in comparison with basal ganglia and prefrontal cortex measured with an improved dinitrophenylhydrazine assay. $J$ Neurochem. 1998;70(1):268-275.

14. Zhang J, Perry G, Smith MA, Robertson D, Olson SJ, Graham DG, et al. Parkinson's disease is associated with oxidative damage to cytoplasmic DNA and RNA in substantia nigra neurons. Am J Pathol. 1999;154(5):1423-1429.

15. Barthwal MK, Srivastava N, Dikshit M. Role of nitric oxide in a progressive neurodegeneration model of Parkinson's disease in the rat. Redox Rep. 2001;6(5):297-302.

16. Okuno T, Nakatsuji Y, Kumanogoh A, Moriya M, Ichinose $\mathrm{H}$, Sumi H, et al. Loss of dopaminergic neurons by the induction of inducible nitric oxide synthase and cyclooxygenase-2 via CD 40: relevance to Parkinson's disease. J Neurosci Res. 2005;81(6):874-882.

17. Bredt DS. Endogenous nitric oxide synthesis: Biological functions and pathophysiology. Free Radic Res. 1999;31(6):577-596.

18. Conti A, Miscusi M, Cardali S, Germano A, Suzuki H, Cuzzocrea S, et al. Nitric oxide in the injured spinal cord: synthases cross-talk, oxidative stress and inflammation. Brain Res Rev. 2007;54(1):205-218.
19. Hague S, Peuralinna T, Eerola J, Hellstrom O, Tienari PJ, Singleton AB. Confirmation of the protective effect of iNOS in an independent cohort of Parkinson disease. Neurology. 2004;62(4):635-636.

20. Szabo C, Ischiropoulos H, Radi R. Peroxynitrite: Biochemistry, pathophysiology and development of therapeutics. Nat Rev Drug Discov. 2007;6(8):662-680.

21. Singh S, Das T, Ravindran A, Chaturvedi RK, Shukla Y, AgarwalAK, et al. Involvement of nitric oxide in neurodegeneration: a study on the experimental models of Parkinson's disease. Redox Rep. 2005;10(2):103-109.

22. Hunot S, Boissiere F, Faucheux B, Brugg B, Mouatt-Prigent A, Agid Y, et al. Nitric oxide synthase and neuronal vulnerability in Parkinson's disease. Neuroscience. 1996;72(2):355-363.

23. Broom L, Marinova-Mutafchieva L, Sadeghian M, Davis JB, Medhurst AD, Dexter DT. Neuroprotection by the selective iNOS inhibitor GW274150 in a model of Parkinson disease. Free Radic Biol Med. 2011;50(5):633-640. http:// dx.doi.org/10.1016/j.freeradbiomed.2010.12.026.

24. Ochiai T, Ohno S, Soeda S, Tanaka H, Shoyama Y, Shimeno H. Crocin prevents the death of rat pheochromocytoma (PC-12) cells by its antioxidant effects stronger than those of a-tocopherol. Neurosci Lett. 2004;362(1):61-64. http://dx.doi.org/10.1016/j.neulet.2004.02.067.

25. Assimopoulou AN, Sinakos Z, Papageorgiou VP. Radical scavenging activity of Crocus sativus L. extract and its bioactive constituents. Phytother Res. 2005;19(11):997-1000.

26. Soeda S, Ochiai T, Shimeno H, Saito H, Abe K, Tanaka H, et al. Pharmacological activities of crocin in saffron. J Nat Med. 2007;61(2):102111. http://dx.doi.org/10.1007/s11418-006-01209.

27. Nam KN, Park YM, Jung HJ, Lee JY, Min BD, Park SU, et al. Anti-inflammatory effects of crocin and crocetin in rat brain microglial cells. Eur $J$ Pharmacol. 2010;648(1-3): 110-116. 
28. Hemshekhar M, Santhosh SM, Sunitha K, Thushara RM, Kemparaju K, Rangappa KS, et al. A dietary colorant crocin mitigates arthritis and associated secondary complications by modulating cartilage deteriorating enzymes, inflammatory mediators and antioxidant status. Biochimie. 2012;94(12):2723-2733. http:// dx.doi.org/10.1016/j.biochi.2012.08.013.

29. Rajaei R, Hadjzadeh MA, Nemati H, Hosseini M, Ahmadi M, Shafiee S. Antihyperglycemic and antioxidant activity of crocin in streptozotocin-induced diabetic rats. J Med Food. 2013;16(3):206-210. http://dx.doi.org/10.1089/ jmf.2012.2407.

30. Lee IA, Lee JH, Baek NI, Kim DH. Antihyperlipidemic effect of crocin isolated from the fructus of Gardenia jasminoides and its metabolite Crocetin. Biol Pharm Bull. 2005;28(11):2106-2110.

31. He Sy, Qian ZY, Tang FT, Wen N, Xu GL, Sheng L. Effect of crocin on experimental atherosclerosis in quails and its mechanisms. Life Sci. 2005;77(8):907-921.

32. Naghizadeh B, Mansouri MT, Ghorbanzadeh B, Farbood Y, Sarkaki A. Protective effects of oral crocin against intracerebroventricular streptozotocin-induced spatial memory deficit and oxidative stress in rats. Phytomedicine. 2013;20(6):537-542. http://dx.doi.org/ 10.1016/j.phymed.2012.12.019.

33. Zheng YQ, Liu JX, Wang JN, Xu L. Effects of crocin on reperfusion-induced oxidative/nitrative injury to cerebral microvessels after global cerebral ischemia. Brain Res. 2007;1138:86-94.

34. Rajaei Z, Alaei H, Nasimi A, Amini H, Ahmadiani A. Ascorbate reduces morphine-induced extracellular DOPAC level in the nucleus accumbens: A microdialysis study in rats. Brain Res. 2005;1053(1-2):62-66.

35. Paxinos G, Watson C. The rat brain in stereotaxic coordinates. $5^{\text {th }}$ ed. Amsterdam, London: Elsevier Academic; 2005.

36. Fujita M, Nishino H, Kumazaki M, Shimada S, Tohyama M, Nishimura T. Expression of dopamine transporter mRNA and its binding site in fetal nigral cells transplanted into the striatum of 6-OHDA lesioned rat. Mol Brain Res. 1996;39(1-2):127-136.
37. Sharma JB, Sharma A, Bahadur A, Vimala N, Satyam A, Mittal S. Oxidative stress markers and antioxidant levels in normal pregnancy and pre-eclampsia. Int $J$ Gynaecol Obstet. 2006;94(1):23-27.

38. Perese DA, Ulman J, Viola J. Ewing SE, Bankiewicz KS. A 6-hydroxydopamine-induced selective parkinsonian rat model. Brain Res. 1989;494(2):285-293.

39. Deumens R, Blokland A, Prickaerts J. Modeling Parkinson's disease in rats: An evaluation of 6-OHDA lesions of the nigrostriatal pathway. Exp Neurol. 2002;175(2):303-317.

40. Schwarting RK, Huston JP. Behavioral and neurochemical dynamics of neurotoxic mesostriatal dopamine lesions. Neurotoxicol. 1997;18(3):689-708.

41. Ungerstedt U. Postsynaptic supersensitivity after 6-hydroxy-dopamine induced degeneration of the nigro-striatal dopamine system. Acta Physiol Scand Suppl. 1971;367:69-93.

42. Shapiro RM, Glick SD, Camarota NA. A two-population model of rat rotational behavior: effects of unilateral nigrostriatal 6-hydroxydopamine on striatal neurochemistry and amphetamine-induced rotation. Brain Res. 1987;426(2):323-331.

43. Schwarting RKW, Hudson JL. The unilateral 6-OHDA injection lesion model in behaviour brain research: analysis of functional deficit, recovery and treatment. Prog Neurobiol. 1996;50(2-3):275-331.

44. Hritcu L, Ciobica A, Artenie V. Effects of rightunilateral 6-hydroxydopamine infusioninduced memory impairment and oxidative stress: Relevance for Parkinson's disease. Cent Eur $J \quad$ Biol. 2008;3(3):250-257. http://dx.doi. org/10.2478/s11535-0o8-0023-8.

45. Haleagrahara N, Siew CJ, Ponnusamy K. Effect of quercetin and desferrioxamine on 6-hydroxydopamine (6-OHDA) induced neurotoxicity in striatum of rats. $J$ Toxicol Sci. 2013;38(1):25-33.

46. Mansouri MT, Farbood Y, Sameri MJ, Sarkaki A, Naghizadeh B, Rafeirad M. Neuroprotective effects of oral gallic acid against oxidative stress induced by 6-hydroxydopamine in rats. Food Chem. 2013;138(2-3):1028-1033. http://dx.doi. org/10.1016/j.foodchem.2012.11.022. 
Original Article | Crocin and 6-OHDA-induced oxidative/nitrosative damage

47. Sarre S, Yuan H, Jonkers N, Van Hemelrijck A, Ebinger G, Michotte Y. In vivo characterization of somatodendritic dopamine release in the substantia nigra of 6-hydroxydopamine-lesioned rats. J Neurochem. 2004;90(1):29-39.

48. Di Matteo V, Pierucci M, Benigno A, Crescimanno G, Esposito E, Di Giovanni G. Involvement of nitric oxide in nigrostriatal dopaminergic system degeneration: a neurochemical study. Ann $N$ YAcad Sci. 2009;1155:309-315. http://dx.doi. org/10.1111/j.1749-6632.2008.03678.x.

49. Gupta SP, Patel S, Yadav S, Singh AK, Singh $\mathrm{S}$, Singh MP. Involvement of nitric oxide in maneb- and paraquat-induced Parkinson's disease phenotype in mouse: Is there any link with lipid peroxidation? Neurochem Res. 2010;35(8):1206-1213. http://dx.doi. org/10.1007/s11064-010-0176-5.

50. Jachymova M, Masters BS, Horky K, Zima T, Martasek P. Nitric oxide synthase, typical flavohemoproteins and their complicated enzymology. Cas Lek Cesk. 2006;145(7):526531.
51. Gupta SP, Yadav S, Singhal NK, Tiwari MN, Mishra SK, Singh MP. Does restraining nitric oxide biosynthesis rescue from toxins-induced parkinsonism and sporadic Parkinson's disease? Mol Neurobiol. 2014;49(1):262-275. http://dx.doi.org/10.1007/s12035-013-8517-4.

52. Singh S, Kumar S, Dikshit M. Involvement of the mitochondrial apoptotic pathway and nitric oxide synthase in dopaminergic neuronal death induced by 6-hydroxydopamine and lipopolysaccharide. Redox Rep. 2010;15(3):115-122. http://dx.doi. org/10.1179/174329210X12650506623447.

53. Guo S, Yan J, Yang T, Yang X, Bezard E, Zhao B. Protective effects of green tea polyphenols in the 6-OHDA rat model of Parkinson's disease through inhibition of ROS-NO pathway. Biol Psychiatry. 2007;62(12):1353-1362. 